\title{
Indicadores de Balance Social en el sector cooperativista
}

\section{Social Balance Indicators in the cooperative sector}

1 Jacqueline Carolina Sánchez Lunavictoria https://orcid.org/0000-0002-3059-2823 Escuela Superior Politécnica de Chimborazo, Facultad de Administración de Empresas, Ecuador carolina.sanchez@espoch.edu.ec

2 Gina Patricia Cuadrado Sánchez Universidad Católica de Cuenca, Unidad Académica de Administración, Ecuador gcuadrado@ucacue.edu.ec

4 Jorge Vinicio Cárdenas Muñoz (iD) https://orcid.org/0000-0001-8663-4677 Universidad Católica de Cuenca extensión Cañar, Unidad Académica de Informática, Ciencias de la Computación e Innovación Tecnológica, Ecuador jvcardenasm@ucacue.edu.ec

Artículo de Investigación Científica y Tecnológica Enviado: 08/11/2021

VISIONARIO DIGITAL, es una Revista Científica, Trimestral, que se publicará en soporte electrónico tiene como misión contribuir a la formación de profesionales competentes con visión humanística y crítica que sean capaces de exponer sus resultados investigativos y científicos en la misma medida que se promueva mediante su intervención cambios positivos en la sociedad. https://visionariodigital.org 
Palabras

claves: balance

social,

cooperativismo,

indicadores,

principios

cooperativos,

bien común,

responsabilidad

social

\section{Keywords:}

social balance, cooperatives, indicators, cooperative principles, common good, social responsibility.
Resumen

Introducción. Las cooperativas de ahorro y crédito constituyen un componente fundamental en la sociedad ya que contribuyen de manera significativa a la construcción de una economía participativa, democrática y solidaria. No obstante, el compromiso de asumir la responsabilidad producto de su accionar es deber de toda organización, superando el enfoque filantrópico y altruista con el que muchas veces se confunde este término. Es así como el objetivo que se plantea esta investigación es aplicar indicadores que permitan evaluar la gestión de responsabilidad social, alineados al cumplimiento de los principios cooperativos. Metodología. La investigación es de tipo no experimental de corte transversal descriptiva, una vez que los hallazgos han sido presentados estos se describen y se sintetizan en base a una interpretación. Resultados. La aplicación de 23 indicadores de balance social, articulados a los principios cooperativos permitieron evaluar la gestión de responsabilidad social de la organización y proporcionar información que sirva de base para la toma de decisiones. Conclusiones. A partir de los resultados obtenidos se puede concluir que en la organización existen muy pocas deficiencias a nivel de la gestión social que deben ser atendidas ya que en gran parte los resultados registran resultados satisfactorios.

\section{Abstract}

Introduction. Savings and credit cooperatives constitute a fundamental component in society since they contribute significantly to the construction of a participatory, democratic and solidary economy. However, the commitment to assume responsibility because of their actions is the duty of every organization, overcoming the philanthropic and altruistic approach with which this term is often confused. The objective of this research is to apply indicators that allow evaluating social responsibility articulated to the fulfillment of cooperative principles. Methodology. The research is of a non-experimental, descriptive cross-sectional type, once the findings have been presented, they are described and synthesized based on an interpretation. Results. The application of 23 social balance indicators, articulated with the cooperative principles, allowed evaluating the organization's social responsibility management and 
providing information that served as the basis for decision-making. Conclusions. From the results obtained, it can be concluded that there are very few deficiencies at the level of social management that must be addressed, since in large part the results register satisfactory results.

\section{Introducción}

Los indicadores de balance social se constituyen en una valiosa herramienta de gestión que les permite a las instituciones evaluar cuantitativa y cualitativamente el cumplimiento de su gestión social tanto en su área interna como externa durante un período determinado.

Esta herramienta además constituye un importante soporte para la administración puesto que facilita la toma de decisiones en el proceso de mejoramiento de la calidad de los servicios y fundamentalmente en lo que respecta a su gestión social (Sánchez, 2014).

En suma, el informe de balance social cooperativo permite: 1) Reflejar el cumplimiento de sus objetivos ligados al cumplimiento de los principios cooperativos; 2) Proponer acciones para mejorar su accionar en el contexto en el que está inserta; 3) Facilitar la toma de decisiones 4) Conocer las fortalezas y debilidades, facilitando a los organismos del gobierno la supervisión y control 5) Dotar de valor a sus operaciones con un sentido de pertinencia social (Sánchez, 2015).

En este orden de ideas se puede decir que al darles un enfoque social a cada uno de los indicadores se provee a la institución de una herramienta de gestión que facilitará su autoevaluación a la vez que le permite dar cuenta a sus grupos de interés sobre su accionar en relación con el cumplimiento de los principios cooperativos.

\section{Antecedentes históricos del Balance Social}

Se sabe que el origen del primer Balance Social tuvo su origen en Estados Unidos de América como mecanismo para propiciar la conciencia social por sobre el capital y la acumulación de riqueza. Se cree que fue la empresa Singer quien en la década de los setenta publicó su primer Balance Social. Esta herramienta se propagó en Europa, en países como Alemania y Francia, y luego en todo el mundo. A partir de allí, se ha establecido como un referente para ciertos países y corporaciones internacionales (Red de Empresas para el Desarrollo Sostenible (DERES, 2015).

Es en la década de los ochenta donde varias modelos de Balance Social se dan a conocer y son adoptados por varias organizaciones gubernamentales y no gubernamentales especialmente en Europa. 
En algunos países de Latinoamérica, el Balance Social apareció casi al mismo tiempo que en el resto del globo. Cabe destacar que aun en esa época no se profundizó en su concepción ni en promover su implementación. Particularmente fue en Chile y en Brasil donde se cree se dieron las primeras iniciativas en lo que respecta a Sur América (Sánchez, 2014).

Para Curto (2004) (citado por Asencio Gallardo, 2015), en Iberoamérica las principales instituciones públicas y privadas de países como Chile, Ecuador, Brasil, Colombia, México entre otros publican anualmente su Balance Social.

En las últimas décadas, el tema y la adopción de la Responsabilidad Social Empresarial se ha establecido de forma general, aunque no obligatoria en casi todo tipo de empresas (Buchholtz y Rosental, 2011).

\section{Definición de Balance Social Cooperativo}

Hoy en día la sociedad tiende a ver con mejores ojos a aquellas empresas, que también colaboran para el desarrollo social y sostenible de su comunidad. Es aquí donde la importancia de que las organizaciones informen sobre los impactos de su accionar a su comunidad resulta relevante y muy valorado (Sánchez, 2014).

Si todas las organizaciones pudiesen ver más allá de su objetivo lucrativo y le devolvieran a la sociedad parte de lo que reciben de ella, se tendría no solo empresas socialmente responsables sino comprometidas con el bien común (Sánchez, 2014).

Pese a que las cooperativas en el contexto latinoamericano desarrollan una importante labor social atendiendo las necesidades de sus asociados y comprometiéndose con la solución de los problemas de su comunidad, carecen en su mayoría de un instrumento de gestión que le permita garantizar la mejor utilización de los recursos y cumplimiento de sus principios cooperativos (Alfonso et al., 2008).

Es así como el Balance Social Cooperativo se constituye en el instrumento ideal para la toma de decisiones, facilitando la gestión no solo en el ámbito administrativo sino también en el ámbito social. Además, como se mencionó en líneas anteriores, la comunidad y particularmente los clientes demuestran mayor apego por aquellas empresas que son socialmente responsables, no es coincidencia entonces que muchas empresas hoy en día empleen la responsabilidad social como estrategia comercial y publicitaria. Para Colina y Senior (2008), Tusa (2020), el Balance Social se determina como un elemento de diagnóstico, planificación, comunicación y control, permitiendo el establecimiento de unos objetivos sociales y una mejor gestión de los aspectos internos y externos derivados de su actividad, lo cual se reflejará en mayor bienestar para la comunidad y en un incremento de la rentabilidad del negocio. 
Por su parte Oliveros (2016), define al balance social como el estado que permite conocer de manera sistemática las acciones de responsabilidad social de la organización, midiéndola cualitativa y cuantitativamente de forma permanente y sistemática, para identificar los programas que favorezcan el desarrollo efectivo de la acción social e informar a los agentes internos y externos.

Otro aporte es el que presenta Luque et al. (2019), quien define al Balance Social como el instrumento que reúne y sistematiza la información de áreas como la medioambiental, económica y social. Así mismo González y San Bartolomé (2008) (citado en Luque, 2019), lo define como:

una herramienta de la gestión socioeconómica que permite a las cooperativas medirse y mostrar su eficiencia a los asociados [...] y a todos los demás grupos de interés que están impactando por su accionar en relación con el cumplimiento de su propia esencia o identidad, es decir los valores y principios cooperativos. (p.34)

Al respecto de la importancia de esta herramienta de gestión, Mugarra (2001) expresa que:

gracias a este instrumento, las cooperativas contarán con un medio adecuado para medir el grado de acercamiento o alejamiento que tengan con respecto al cumplimiento de su misión como organización cooperativa, a la luz de sus principios y valores cooperativos. (p.10)

En este orden de ideas Capron y Leseul (1997) (citado en Ribas, 2001) manifiesta que "medir la acción de nuestras empresas es una necesidad. Necesidad de evaluación, necesidad de transparencia, necesidad de comunicación" (p.29).

En resumen, según la Alianza Cooperativa Internacional (ACI, 2014), se podría decir que el Balance Social es:

- Una herramienta que permite medir del impacto social de una cooperativa

- Una herramienta que propicia el cambio sistemático a la responsabilidad social

- Una herramienta estratégica de análisis y evaluación sistemática

- Un instrumento de información a la sociedad sobre el accionar de una empresa

\section{Principios cooperativos}

Los principios cooperativos son lineamientos generales a través de los cuales las cooperativas plasman sus valores. Su origen se considera a partir de las normas establecidas por la Rochdale Equitable Pioneers Society, que fue fundada en 1844 en 


\section{VPDigitital}

Inglaterra como una cooperativa de consumo, siendo la primera en distribuir entre sus socios los excedentes generados por la actividad (ACI, 2014).

Actualmente es la Alianza Cooperativa Internacional (ACI, 2014), es quien mantiene vigente estos principios, siendo estos:

1. Asociación voluntaria y abierta

2. Control democrático por los asociados

3. Participación económica de los asociados

4. Autonomía e independencia

5. Educación, capacitación e información

6. Cooperación entre cooperativas

7. Preocupación por la comunidad.

En el campo del cooperativismo, la propuesta presentada por Fernández et al. (1998), sin duda constituye un aporte valioso en lo que respecta al estudio del Balance Social Cooperativo, particularmente en Latinoamérica, donde resultan escazas estas iniciativas. El sistema de indicadores que presentan los autores se configura a partir de su alineación a cada principio cooperativo como mecanismo que permita medir el cumplimiento de la responsabilidad social. Si bien es cierto esta obra constituye un aporte adecuado a la realidad argentina, es su flexibilidad lo que le permite adaptarse a las particularidades de otros contextos, además porque los principios declarados son considerados universales para el ámbito cooperativista. Es así como para el desarrollo de esta investigación se adopta esta propuesta para su aplicación.

\section{Metodología}

La investigación es de tipo no experimental de corte transversal descriptiva, una vez que los hallazgos han sido presentados estos se describen y se sintetizan en base a una interpretación.

Al mismo tiempo puede decirse que la investigación es de tipo explicativa, porque se explican y analizan cada uno de los resultados.

\section{Resultados}

\section{Indicadores de Balance Social alineados al cumplimiento de los principios} cooperativos. Caso Cooperativa de Ahorro y Crédito Educadores de Chimborazo.

Los resultados que se exponen forman parte de la tesis de maestría desarrollada por el autor principal titulada: Diseño e implementación de indicadores de gestión basados en la metodología de balance social para el mejoramiento de la productividad y la calidad de los servicios en la Cooperativa de Ahorro y Crédito Educadores de Chimborazo, 


\section{VPDigital}

período 2012 - 2013 (Sánchez, 2014).

Principio 1: Asociación voluntaria y abierta

Indicador: Ingreso y egreso de asociados

Este indicador pretender medir que no existan impedimentos para el libre ingreso y egreso de socios.

Cantidad: Total de asociados ingresantes al inicio del ejercicio (mes de enero año en curso)

$$
\begin{gathered}
\text { Porcentaje: } \frac{\text { cantidad }}{\text { cantidad total asociados durante el ejercicio }} \times 100 \\
\frac{34}{185} \times 100
\end{gathered}
$$

$18,37 \%$

Análisis: Del total de socios que ingresaron a la cooperativa durante el año en curso, solo en el mes de enero ingresaron de forma voluntaria un 18,37\%, evidenciándose el cumplimiento del primer principio cooperativo.

Indicador: Causa de egreso de asociados

Las cooperativas deben procurar que no existan impedimentos para que el socio de manera voluntaria se dé de baja.

Cantidad: Total de asociados que se dieron de baja voluntario al inicio del ejercicio

Porcentaje: $\frac{\text { cantidad }}{\text { cantidad total de asociados que se dieron de baja durante el ejercicio }} \times 100$

$$
\frac{5}{85} \times 100
$$

\section{$5,88 \%$}

Análisis: Del total de socios que se dieron de baja voluntaria durante todo el año, solo en el mes de enero egresaron un 5,88\%, evidenciándose que no existen barreras para la baja voluntaria de los socios cuando estos lo requieran.

Principio 2: Control democrático de los asociados-participación y gestión democrática Indicador: Asistencia a asambleas

Asambleas ordinarias 


\section{VPDigitital}

La participación en asambleas permite al asociado ejercer el control democrático. Es en las asambleas donde se toman acciones y se fijan políticas.

Cantidad: Total de asociados asistentes a asamblea ordinaria en calidad de representantes

$$
\text { Porcentaje: } \frac{\text { cantidad }}{\text { cantidad total asociados representantes }} \times 100
$$

$$
\frac{28}{30} \times 100
$$

\section{$93,33 \%$}

Análisis: En la cooperativa de Ahorro y Crédito Educadores de Chimborazo existen 30 representantes que participan en asambleas ordinarias convocadas por la institución. Como se evidencia en el indicador la asistencia y participación de los representantes es casi en su totalidad.

\section{Asambleas extraordinarias}

Cantidad: Total de asociados asistentes a asamblea extraordinaria en calidad de representantes

$$
\begin{aligned}
& \text { Porcentaje: } \frac{\text { cantidad }}{\text { cantidad total asociados representantes }} \times 100 \\
& \frac{29}{30} \times 100 \\
& 96,66 \%
\end{aligned}
$$

Análisis: Igual y como sucede en el indicador anterior la asistencia y participación de los representantes a asambleas extraordinarias es casi en su totalidad.

\section{Indicador: Hombres y mujeres en cargos institucionales}

Las cooperativas deberán asegurar que en sus programas de gestión intervengan igual número de hombres como de mujeres, con este indicador se plantea medir la intervención de ambos géneros en cargos institucionales.

\section{Total mujeres:}

Cantidad: Total de asociados mujeres que ocupan cargos en la institución

$$
\begin{gathered}
\text { Porcentaje: } \frac{\text { cantidad }}{\text { cantidad total de cargos institucionales al cierre del ejercicio }} \times 100 \\
\frac{7}{14} \times 100
\end{gathered}
$$$$
50,00 \%
$$ 


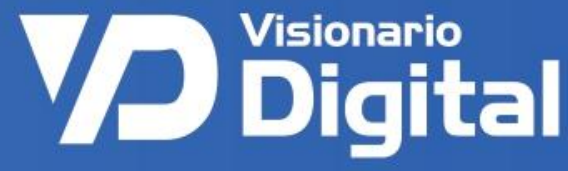

Análisis: En la institución trabajan alrededor de 14 personas de las cuales el $50 \%$ son mujeres lo que evidencia una equilibrada participación de ambos sexos en cargos institucionales.

\section{Hombres y mujeres en cargos institucionales}

Las cooperativas deberán asegurar que en sus programas de gestión intervengan igual número de hombres como de mujeres, con este indicador se plantea medir la intervención de ambos géneros en cargos institucionales.

\section{Total hombres}

Cantidad: Total de asociados hombres que ocupan cargos en la institución

$$
\begin{aligned}
& \text { Porcentaje: } \frac{\text { cantidad }}{\text { cantidad total de cargos institucionales al cierre del ejercicio }} \times 100 \\
& \frac{7}{14} x 100 \\
& 50,00 \%
\end{aligned}
$$

Análisis: En la institución trabajan alrededor de 14 personas de las cuales el 50\% son hombres lo que evidencia una equilibrada participación de ambos sexos en cargos institucionales.

\section{Hombres y Mujeres en cargos institucionales de tipo jerárquico}

Las cooperativas deberán asegurar que en sus programas de gestión intervengan igual número de hombres como de mujeres, con este indicador se plantea medir la participación de ambos géneros en cargos institucionales, pero con poder de mando o jerárquico.

\section{Total mujeres}

Cantidad: Total de asociados mujeres con mando jerárquico

$$
\begin{gathered}
\text { Porcentaje: } \frac{\text { cantidad }}{\text { cantidad total de cargos jerárquicos al cierre del ejercicio }} \times 100 \\
\frac{2}{6} \times 100
\end{gathered}
$$

\section{$33,33 \%$}

Análisis: En la institución existen 6 cargos de tipo jerárquico de los cuales el $33.33 \%$ lo ocupan mujeres, lo que evidencia que no existe una equilibrada participación de ambos sexos en cargos institucionales con poder de mando. 


\section{VPDigitital}

\section{Total Hombres}

Cantidad: Total de asociados hombres con mando jerárquico

$$
\begin{gathered}
\text { Porcentaje: } \frac{\text { cantidad }}{\text { cantidad total de cargos jerárquicos al cierre del ejercicio }} \times 100 \\
\frac{4}{6} \times 100 \\
66,66 \%
\end{gathered}
$$

Análisis: En la cooperativa de Ahorro y Crédito Educadores de Chimborazo existen 6 cargos de tipo jerárquico de los cuales el $66.66 \%$ lo ocupan hombres lo que evidencia que no existe una equilibrada participación de ambos sexos en cargos institucionales con poder de mando.

\section{Información}

Muchas veces depende de la calidad de información que recibe el socio para que se posibilite su participación en asambleas y demás eventos. Por tanto, esta debe ser oportuna y de calidad (Sánchez, 2014).

\section{Indicador: Inversión en Información}

\section{Destinado a empleados y trabajadores}

Cantidad: Monto invertido en información directa destinados a los empleados y trabajadores

$$
\begin{aligned}
& \text { Porcentaje: } \frac{\text { cantidad }}{\text { Monto total invertido en información en el ejercicio }} \times 100 \\
& \frac{3250,34}{276454,96} \times 100
\end{aligned}
$$

\section{$1,17 \%$}

Análisis: Se puede evidenciar que al culminar el ejercicio fiscal la institución invirtió a penas un $1,17 \%$ en mantener informado al cliente interno (empleados y trabajadores) del total destinado para información.

\section{Inversión en Información destinado a socios}

Cantidad: Monto invertido en información directa destinados a socios

$$
\text { Porcentaje: } \frac{\text { cantidad }}{\text { Monto total invertido en información en el ejercicio }} \times 100
$$




\section{VPDigitital}

$$
\frac{214393,42}{276454,96} \times 100
$$

$77,55 \%$

Análisis: Se puede evidenciar que al culminar el ejercicio fiscal la cooperativa invirtió en información destinada a sus socios un $77,55 \%$. Esto evidencia que existe un compromiso de la institución por mantener informados a sus socios.

Inversión en información destinado al público en general

Cantidad: Monto invertido en información directa destinados al público en general

$$
\begin{gathered}
\text { Porcentaje: } \frac{\text { cantidad }}{\text { Monto total invertido en información en el ejercicio }} \times 100 \\
\frac{58811,20}{276454,96} \times 100
\end{gathered}
$$

Análisis: Se puede evidenciar que del total destinado para información un 21, 27\% se destinó para informar a la comunidad. Resulta importante que toda empresa informe a la comunidad sobre los beneficios de su gestión en bien del desarrollo del entorno donde se desenvuelve, esto trae consigo enormes beneficios como aceptación, preferencia, captación de nuevos socios y fidelidad.

Principio 3: participación económica de los asociados - participación y gestión democrática

Indicador: Remuneración a los asociados por sus aportes financieros

Uno de los principales medios de financiamiento que tiene la cooperativa, son los aportes de los socios al capital social o por concepto de préstamos que concede al socio. La remuneración o pago que realice la institución por los conceptos antes mencionados puede favorecer o estimular la participación económica de los asociados.

Remuneración por concepto de aporte al capital social o certificados de aportación

Cantidad: Tasa de interés que ofrece la cooperativa por concepto de aportes al capital social

$$
\begin{gathered}
\text { Porcentaje: } \frac{\text { cantidad }}{\text { tasa pasiva anual que ofrece el mercado }} \\
\frac{8,00 \%}{5.08 \%}
\end{gathered}
$$




\section{VPDigital}

\section{$1,57 \%$}

Análisis: Con este indicador se analiza la remuneración que recibe el socio por concepto de interés por aportaciones al capital. Si el valor resulta inferior a 1, indica que el asociado está perdiendo y que de pronto la competencia le puede ofrecer mejores opciones. Por otro lado, si el resultado es mayor a 1, indica una remuneración al capital cooperativo superior al de mercado.

Indicador: Remuneración que paga el socio por concepto préstamos o créditos

Cantidad: Tasa de interés que cobra la cooperativa por concepto de aportes al capital social

$$
\text { Porcentaje: } \frac{\text { cantidad }}{\text { tasa pasiva media anual que cobra el mercado }}
$$

$$
\frac{15,00 \%}{16,39 \%}
$$

$$
0,91 \%
$$

Análisis: Con este indicador se analiza el pago que tiene que realizar el socio por concepto de préstamos o créditos, comparando con la tasa activa media que se paga en el mercado. Si el valor es mayor que 1 , señala una pérdida relativa para el socio con respecto a alternativas comparables de financiación en el mercado. En cambio, valores inferiores a 1 indican una mejor oportunidad para el socio si recibe financiamiento en la institución.

\section{Indicador: Política social interna de la cooperativa}

El clima social interno influye en la calidad de los servicios que se prestan a los socios y en la manera como proyecta su imagen de cooperativa al exterior. El objetivo de estos indicadores es el de medir cuanto destina y cuanto en realidad invierte la institución en aspectos sociales tanto para socios y empleados.

Cantidad: Monto invertido en servicios sociales al final del ejercicio

$$
\text { Porcentaje: } \begin{gathered}
\text { Monto destinado a servicios sociales al inicio del ejercicio } \\
\frac{8878,13}{10765,56} \\
82,46 \%
\end{gathered}
$$

Análisis: Con este indicador se analiza si se destina una cantidad monetaria a servicios sociales y de esta cuanto realmente se ha utilizado durante el ejercicio. Como se puede observar del $100 \%$ destinado a servicios sociales, se ha empleado un $82,46 \%$. Es 


\section{VPDigital}

importante que la cooperativa planifique de mejor manera como y cuando se va a utilizar estos recursos de tal manera que tantos los socios y empleados se puedan beneficiar de ellos.

\section{Indicador: Seguridad e higiene del trabajo}

Las mejores condiciones de seguridad e higiene disminuyen los accidentes, mejoran la calidad de vida de los empleados y los predisponen a brindan un mejor servicio.

Cantidad: Total de horas laborales perdidas por los empleados durante el ejercicio y producto de accidentes, o enfermedades producto del trabajo.

$$
\text { Porcentaje: } \frac{\text { Cantidad }}{\text { Cantidad de horas laborales en el ejercicio }} \text { X100 }
$$

$$
\frac{25}{1872}
$$

$$
1,33 \%
$$

Análisis: De los resultados podemos afirmar que las condiciones laborales en la organización son idóneas puesto que no se evidencia un mayor número de horas perdidas producto de accidentes o enfermedades a causa del trabajo y de las pocas que se registran son producto de trabajo de campo, y otro tipo de enfermedades como el estrés laboral. El número de horas laboradas y perdidas se obtuvieron de la base de datos del departamento de Talento Humano.

\section{Principio 4: Autonomía e independencia- independencia financiera}

Indicador: Independencia financiera con relación a los fondos propios y activos totales. Es importante para conocer qué parte del activo de la empresa está financiado con fondos propios.

Cantidad: Fondos propios

$$
\begin{gathered}
\text { Porcentaje: } \frac{\text { cantidad }}{\text { Total Activo }} \\
\frac{2900.456,34}{20100.000,00}
\end{gathered}
$$

$14,42 \%$

Análisis: Se puede evidenciar que la cooperativa cuenta con una total independencia financiera, puesto que su activo total no depende de las aportaciones que realizan sus socios. 


\section{VPDigitital}

\section{Autonomía}

\section{Indicador: Intervención de otras organizaciones en puestos jerárquicos en la cooperativa}

Este indicador provee de información sobre algún grado de limitación o afectación a la autonomía e independencia en lo que respecta a la toma de decisiones de la institución.

Cantidad: Cargos ocupados de tipo jerárquico en la cooperativa por representantes de otros organismos o cooperativas socias.

$$
\begin{gathered}
\text { Porcentaje: } \frac{\text { cantidad }}{\text { Total de cargos cooperativos con nivel jerárquico }} \times 100 \\
\frac{0}{8} \times 100 \\
0 \%
\end{gathered}
$$

Análisis: Con el resultado de este indicador se evidencia que no está en riesgo la autonomía de la cooperativa puesto que no existen cargos jerárquicos ocupados por otros representantes ajenos a la misma.

Principio 5: Educación capacitación e información- política educativa de la cooperativa Indicador: Inversión en formación, entrenamiento e información

La inversión en educación, entrenamiento e información conforman el apoyo y financiamiento a actividades educativas para el beneficio de los socios y empleados de la cooperativa.

Cantidad: Monto invertido en el ejercicio a actividades de educación, capacitación e información al final del ejercicio.

$$
\text { Porcentaje: } \frac{\text { cantidad }}{\text { MOnto total destinado a actividades de educacion al inicio del ejercicio }}
$$

$$
\frac{31317,58}{31317,58}
$$

$100 \%$

Análisis: De los resultados se evidencia que se usó el $100 \%$ del total destinado para eventos de educación y capacitación tanto de socios como de empleados, se puede decir además que esta es una de las actividades que más se practica al interior de la cooperativa.

Inversión en educación, destinada a socios de la cooperativa

El monto invertido en educación, capacitación e información comprende apoyo y financiamiento a actividades educativas para el beneficio solo de los socios. 


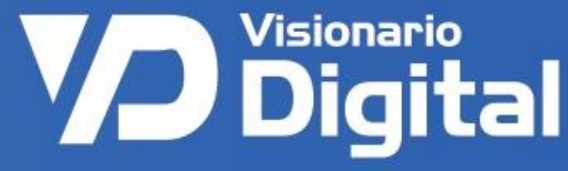

Cantidad: Monto invertido en el ejercicio a actividades de educación, capacitación e información solo para socios.

$$
\begin{gathered}
\text { Porcentaje: } \frac{\text { cantidad }}{\text { MOnto total destinado a educacion destinado a inicio del ejercicio }} \\
\frac{25321,64}{31317,58} \times 100 \\
80,85 \%
\end{gathered}
$$

Análisis: Del total de recursos monetarios destinados a actividades de educación y capacitación un $80 \%$ fue destinado a capacitar a los socios de la cooperativa, que se justifica por el número de asistentes y capacitadores para cubrir toda la demanda y demás gastos de logística.

\section{Inversión en educación, destinada a empleados y trabajadores}

El monto invertido en educación, capacitación e información comprende apoyo y financiamiento a actividades educativas para el beneficio de los empleados de la cooperativa.

Cantidad: Monto invertido en el ejercicio a actividades de educación, capacitación e información solo para empleados y trabajadores.

$$
\begin{gathered}
\text { Porcentaje: } \frac{\text { cantidad }}{\text { MOnto total destinado a actividades de educacion al inicio del ejercicio }} \\
\frac{5995,94}{31317,58} \times 100
\end{gathered}
$$

$$
19,15 \%
$$

Análisis: Del total de recursos monetarios destinados a actividades de educación y capacitación un $19,15 \%$ fue destinado a capacitar a los empleados y trabajadores de la cooperativa, que se justifica porque son apenas 14 empleados en total. Lo importante es que se empleó el $100 \%$ de este recurso.

\section{Principio 6: Cooperación entre cooperativas Inter cooperación}

\section{Operaciones comerciales o financieras con otras cooperativas}

Se cree que las cooperativas cumplen con este principio cuando procuran acuerdos de mutua colaboración y ejecutan trabajos en común con otras instituciones cooperativistas.

Indicador: Préstamos otorgados a otras cooperativas 


\section{VPDigitital}

Cantidad: Valor total de créditos concedidos a cooperativas, al cierre del ejercicio cancelados o no cancelados

$$
\text { Porcentaje: } \frac{\text { cantidad }}{\text { MOnto total de préstamos otrogados en el ejercicio }} \times 100
$$

$$
\frac{0}{17125127,75}
$$

$0 \%$

Análisis: La cooperativa no ha realizado préstamos a otras cooperativas, el 100\% está destinado a cubrir demandas de créditos o préstamos de sus socios.

Indicador: Préstamos recibidos de otras cooperativas

Cantidad: Monto total empleado para financiamiento al cierre del ejercicio

Porcentaje: $\frac{\text { cantidad }}{\text { Monto total de financiamiento externo en el ejercicio }} \times 100$

$$
\frac{799843,72}{799843,72}
$$

$100 \%$

Análisis: EL 100\% de financiamiento lo recibe de parte otras entidades financieras con las que la cooperativa mantiene acuerdos y como se evidencia el 100\% del financiamiento fue empleado para cubrir actividades propias de su gestión.

Principio 7: Preocupación por la comunidad - política social externa de la cooperativa Indicador: Aportes para el desarrollo de la comunidad

Las cooperativas consiguen contribuir al desarrollo de su sociedad con actividades y contribuciones que propendan al desarrollo social, medioambiental, y cultural.

Cantidad: Monto invertido en aportes para el desarrollo social realizadas en el ejercicio.

Porcentaje: $\frac{\text { cantidad }}{\text { Monto total destinado para el desarollo comunitario al inicio del ejercicio }} \times 100$

$$
\frac{0}{0}
$$

Análisis: Como se evidencia; lamentablemente no se han desarrollado ningún tipo de proyectos o programas medioambientales, asuntos culturales ni deportivos, que beneficien a la comunidad, por tanto, no se estaría cumpliendo este principio. 


\section{Conclusiones}

- Pese al reconocimiento de la importancia que representa la elaboración de un Balance Social como herramienta que permite evaluar y mejorar la gestión social de las organizaciones ha sido muy poco tratado y aplicado en las organizaciones.

- El sistema de indicadores alineados a cada uno de los principios cooperativos permitió realizar una medición y análisis de la gestión social de la organización.

- De los resultados alcanzados se concluye que existen muy pocas deficiencias a nivel de la gestión social que deben ser atendidas por la institución particularmente ya que en gran parte los resultados registran niveles satisfactorios.

\section{Referencias bibliográficas}

Alfonso, A. Rivera, R. J., Labrador, M. C., Labrador, O. (2008). Responsabilidad y balance social en las empresas cooperativas. Revista de Ciencias Sociales, 14(1), 09-19.

Asencio, G. G. (2015). El Balance Social como una herramienta válida para representar la Responsabilidad Social en las Empresas Chilenas. Revista Ciencia Unemi, $8(16), 87-96$.

Alianza Cooperativa Internacional [ACI]. (2014). Balance Social Cooperativo: Certificación de cooperativas de las américas en RSC. https://www.aciamericas.coop/Balance-Social-Cooperativo-2394.

Buchholtz, R. y Rosental, S. (2011). Responsabilidad Social y Ética en los negocios. Ediciones Oxford.

Capron, M., y Leseul, G., (1997). Pour un bilan sociétal des entreprises. Recma, 266, 2841.

Colina, J., y Senior, A. (2008). Balance social. Instrumento de análisis para la gestión empresarial responsable. Multiciencias, 8(2),71-77.

Curto G. M. (2004). La responsabilidad social interna de las empresas interna". Universidad de Navarra, IESE, Business School. Cuadernos de la Cátedra "la Caixa" de Responsabilidad Social de la Empresa y Gobierno Corporativo, 16(4), 32-35

Red de Empresas para el Desarrollo Sostenible [DERES]. (2015). Manual para la preparación e implementación del Balance Social en el Uruguay. https://deres.org.uy/wp-content/uploads/2020/05/Manual-DERESPreparaci\%C3\%B3n-para-elaborar-el-Balance-Social.pdf 


\section{VPDigital}

Fernández L., Geba N., Montes V., Schaposnik R. (1998). Balance Social Cooperativo Integral. disponible en https://core.ac.uk/download/pdf/296377697.pdf

González, L., y San Bartolomé, J. (2008). Balance Social Cooperativo: Una construcción en Construcción. Argentina: Centro de Acción, Desarrollo e Investigación en Cooperativas y Mutuales. https://www.aciamericas.coop/Balance-SocialCooperativo-Una

Luque, A., Peñaherrera, J., \& Ordoñez, J. (2019). Comparación de los resultados del balance social como herramienta de gestión: los casos de Cacec y Chibuleo. Revista Chakiñan de ciencias sociales y humanidades, (7), 104-117.

Mugarra, E. A. (2001). Responsabilidad y balance social hoy en día: un reto para las cooperativas. CIRIEC-España. Revista de Economía Pública, Social y Cooperativa, (39), 25-50

Oliveros, M. (2016). El balance Social como Herramienta de Responsabilidad Social Empresarial: Una Aproximación Teórica. Sapienza Organizacional, 3, (6), 93-106

Ribas B. M. (2001). El balance social como instrumento para la evaluación de la acción social en las entidades no lucrativas. CIRIEC-España, Revista de Economía Pública, Social y Cooperativa, (39),115-147

Sánchez J. (2014). Diseño e implementación de indicadores de gestión basados en la metodología de balance social para el mejoramiento de la productividad y la calidad de los servicios en la cooperativa de ahorro y crédito educadores de Chimborazo. (Tesis de Maestría). Universidad Nacional de Chimborazo Ecuador.

Tusa, Verónica. (2020). El balance social y los principios cooperativos en la Cooperativa de Ahorro y Crédito Vencedores de Tungurahua. (Tesis de Maestría). Universidad Técnica de Ambato.

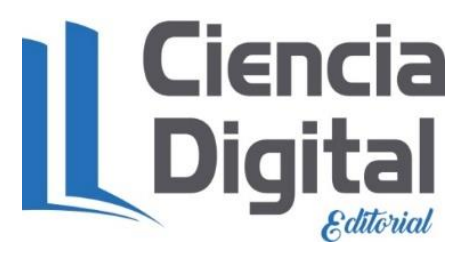




\section{VPDigital}

El artículo que se publica es de exclusiva responsabilidad de los autores y no necesariamente reflejan el pensamiento de la Revista Visionario Digital.

\section{\Ciencia}

El artículo queda en propiedad de la revista y, por tanto, su publicación parcial y/o total en otro medio tiene que ser autorizado por el director de la Revista Visionario Digital.
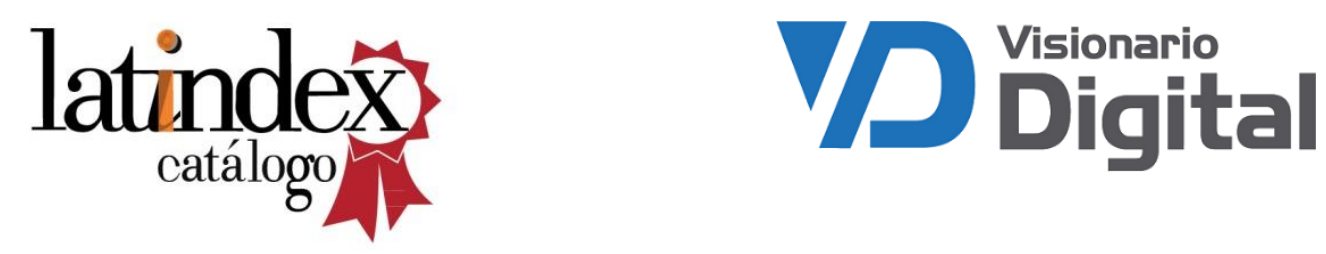

Indexaciones

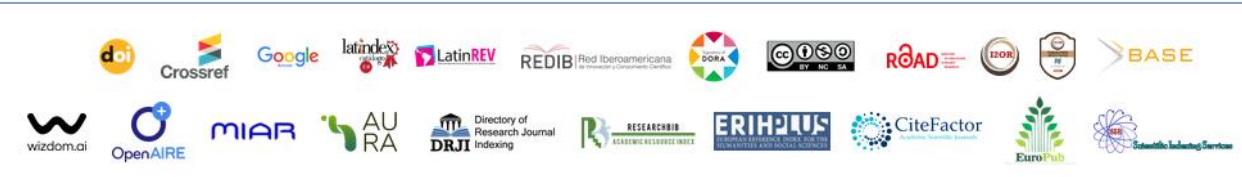

